\title{
COMMUNICATION
}

\section{Second cancer primitif : carcinome cuniculatum de la mandibule dans un contexte de cancer prostatique métastatique}

\section{Broly $E^{1,2}$, Barthélémy $P^{3,4}$, Ciftci $S^{4,5}$, Borel $C^{6}$, Dubourg S1, Guihard $\mathbf{S}^{7}$, Marcellin $L^{8}$, Lutz JC4,9,10, Bornert F1,10,11}

1. Unité de Pathologie-Chirurgie buccale, Pôle de Médecine et de Chirurgie buccodentaires, Hôpitaux Universitaires de Strasbourg 2. UFR d'Odontologie, Université de Reims Champagne-Ardenne

3. Département d'Oncologie, Pôle d'Oncologie-Hématologie, Hôpitaux Universitaires de Strasbourg

4. Faculté de Médecine, Université de Strasbourg

5. Service d'ORL et de Chirurgie cervico-faciale, Pôle Tête et Cou, Hôpital de Hautepierre, Hôpitaux Universitaires de Strasbourg

6. Service d'Oncologie médicale, Centre Paul Strauss, Strasbourg

7. Service de Radiothérapie, Centre Paul Strauss, Strasbourg

8. Département de Pathologie, Hôpitaux Universitaires de Strasbourg ( HUS )

9. Service de Chirurgie Plastique, Esthétique, Reconstructrice, Maxillo-Faciale et Stomatologie - HUS Strasbourg

10. INSERM "Osteoarticular and Dental Regenerative Nanomedicine", UMR 1109, Faculté de Médecine, Strasbourg

11. Faculté de Chirurgie-Dentaire, Université de Strasbourg

\section{Introduction}

Tandis que l'incidence des cancers augmente, la survie des patients cancéreux s'améliore. En 2008, la population ayant un antécédent de cancer en France a été estimée à 3 millions. Dans ce contexte, prévenir la survenue d'un second cancer primitif (SCP = un nouveau cancer autre qu'une récidive locale ou une métastase apparaissant au-delà de 2 mois) est un nouveau défi de santé publique à relever. Les études dédiées à l'identification des facteurs de risque de SCP ne permettent pas encore une prédiction précise.

La détection des SCP nécessite ainsi une attention particulière de l'équipe oncologique et des praticiens du réseau de spécialistes d'organe comme le chirurgien oral.

\section{Observation}

Un patient de 78 ans suivi pour un carcinome prostatique d'emblée métastatique osseux et devenu résistant à la castration (CPRC) décrit des douleurs dentaires névralgiques insomniantes et très invalidantes. Devant une suspicion d'infection odontogène le patient bénéficie de plusieurs antibiothérapies infructueuses et finalement la dent 37 a été extraite. Un retard de cicatrisation de 2 mois de l'alvéole d'extraction a justifié une biopsie intra-alvéolaire mettant en évidence un carcinome épidermoïde envahissant largement le corps mandibulaire et justifiant une hémi-mandibulectomie. Après refus d'une radiothérapie et devant une reprise évolutive ganglionnaire une chimiothérapie par Paclitaxel-Carboplatine-Cetuximab en association avec une hormonothérapie de seconde génération pour le CPRC sont entreprises permettant un contrôle des deux pathologies cancéreuses pendant plus d'un an. L'ensemble des éléments radio-anatomo-cliniques a conclu à un SCP de type carcinome épidermoïde cuniculatum (CC) (2).

\section{Discussion}

Aucun cas de CC ne semble avoir été décrit dans un contexte de CPRC $(2,3)$. Le CC est une tumeur maligne rare de la cavité orale, habituellement retrouvé sur la plante du pied, non associée au tabac et à l'alcool. Les critères cliniques, la lyse osseuse et les éléments anatomo-pathologiques signent son diagnostic avec des atypies cellulaires variables, des boyaux de carcinome organisés en galeries et cryptes contenant des amas de kératine.

(C) The authors, published by EDP Sciences. This is an Open Access article distributed under the terms of the Creative Commons Attribution License 4.0 (http://creativecommons.org/licenses/by/4.0/). 
Le retard diagnostic est fréquent et tout particulièrement en cas de topographie endophytique intra-osseuse (2) ; la radiologie standard peut montrer une lésion radioclaire avec des marges mal définies et une résorption de l'os cortical adjacent qui peuvent passer inaperçus au départ. Le pronostic est assez bon avec la chirurgie d'exérèse seule comme standard (2).

Les différentes études menées dans le monde ne montrent pas de risque global augmenté de SCP au niveau de la sphère ORL en cas CPRC (1). Cependant, Schlieve et al. rapportent un taux de $0,2 \%$ de SCP de la tête du cou de type CE sur une cohorte de 19406 patients cancéreux ; le CPRC était le cancer primitif le plus fréquent $(27,5 \%)$. Sur les 40 patients répertoriés, tous les cancers primitifs confondus, aucun CC n'était rapporté mais la gencive était touchée dans 11 cas (3).

\section{Conclusion}

Ce cas rappelle que toute douleur dentaire persistante chez un patient cancéreux nécessite un bilan approfondi afin d'exclure un SCP ou toute métastase. Par ailleurs, des études sont encore nécessaires pour identifier les facteurs associés aux SCP de la cavité buccale.

fabien.bornert@chru-strasbourg.fr

\section{Références}

(1) Institut National du Cancer. Identifier et prévenir les risques de second cancer primitif chez l'adulte, collection état des lieux et des connaissances. Ouvrage collectif édité par l'INCa, Boulogne-Billancourt, décembre 2013

(2) Pons Y et al. Mandibular cuniculatum carcinoma : a propos of 3 cases and literature review. Head Neck. 2012 Feb ; 34(2) :291-5.

(3) Schlieve T et al. Second primary head and neck cancers after non-head and neck primary cancers. J Oral Maxillofac Surg. 2016; 74(12): 25152520 . 